\title{
Ensaios clínicos com medicamentos no Brasil: uma análise das principais características
}

\section{Clinical trials with drugs in Brazil: an analysis of the main characteristics}

\section{Juliana Carvalho Rocha Alves da Silva' iD}

Rafael Santos Santana" iD

Cecília Menezes Farinasso" iD

Dayde Lane Mendonca da Silvall iD

Helaine Carneiro Capucho, $, 1, *$ iD

Programa de Pós-Graduação em Ciências da Saúde, Universidade de Brasília (UnB), Brasília, DF, Brasil

" Universidade de Brasília (UnB), Brasília, DF, Brasil

\section{* E-mail: helaine.capucho@unb.br}

Recebido: 31 mar 2020

Aprovado: 24 set 2020

\section{RESUMO}

Introdução: Os resultados de ensaios clínicos são utilizados pelas agências regulatórias de todo o mundo para fins de registro e comercialização de medicamentos. A Agência Nacional de Vigilância Sanitária (Anvisa) é a responsável pelos registros de tecnologias em saúde no Brasil e regras para análises técnicas de ensaios clínicos. A Anvisa vem atuando para atualizar seu arcabouço regulatório a respeito de ensaios clínicos com medicamentos no país, para reduzir tempo de análise e harmonizar conforme regras internacionais. Objetivo: Caracterizar os ensaios clínicos de fase III, com medicamentos, realizados no Brasil a partir da publicação da $\mathrm{RDC} \mathrm{n}^{\circ}$ 9, de 20 de fevereiro de 2015, da Anvisa. Método: Estudo exploratório e descritivo realizado em três etapas: (1) análise quantitativa pré e pós $\operatorname{RDC} n^{\circ} 9 / 2015$; (2) análise da população participante de ensaio clínico que embasou registros de medicamentos; (3) caracterização dos ensaios clínicos realizados no Brasil. Resultados: Houve redução em $20 \%$ de ensaios clínicos realizados no Brasil quando se compara o período anterior e posterior à publicação da RDC $n^{\circ}$ 9/2015 da Anvisa; apenas 33\% dos ensaios clínicos que embasaram registros de medicamentos no país foram realizados com população brasileira; os medicamentos sintéticos e biológicos somam $96 \%$ da intervenção estudada nos ensaios clínicos; placebo ainda é muito utilizado como comparador (37\%); a indústria farmacêutica é majoritariamente o patrocinador dos ensaios clínicos (86\%). Conclusões: Diante deste cenário, é imperativo que se fortaleçam as ações de farmacovigilância no Brasil, a fim de conhecer os perfis de efetividade e segurança dos medicamentos após exposição da população brasileira.

PALAVRAS-CHAVE: Ensaio Clínico; Legislação de Medicamentos; Vigilância Sanitária

\section{ABSTRACT}

Introduction: The results of clinical trials (CT) are used by regulatory agencies around the world for the purposes of drug product's registering and marketing. The Brazilian Health Surveillance Agency (Anvisa, in Portuguese) is responsible for the registration of health technologies in Brazil and for creating the rules for the analysis of technical issues in clinical trials. Anvisa has been working to update its regulatory framework regarding clinical trials with drugs in the country, to reduce analysis time and harmonize the normative frame according to international guidelines. Objective: To characterize phase III clinical trials, with drug products, conducted in Brazil from the publication of RDC $n^{\circ}$ 9, on 20 February 2015 by Anvisa. Method: Exploratory and descriptive study, carried out in three stages: (1) quantitative analysis before and after RDC $n^{\circ}$ 9/2015; (2) analysis of the population participating in clinical trials that supported medication records; (3) characterization of the clinical trial performed in Brazil. Results: There was a $20 \%$ reduction in clinical trials conducted in Brazil when compared before and after RDC $n^{\circ} 9 / 2015$ by Anvisa; only 33\% of the clinical trials that supported drug product registrations in Brazil were performed with the Brazilian population; synthetic and biological drugs account for $96 \%$ of the intervention studied in clinical trials; placebo is still widely used as a comparator (37\%); the pharmaceutical industry is mostly the sponsor of the clinical trial (86\%). Conclusions: In view of this scenario, it is imperative to strengthen pharmacovigilance actions in Brazil, in order to learn about the effectiveness and safety profiles of medicines after exposure of the Brazilian population.

KEYWORDS: Clinical Trial; Legislation; Drug Product; Health Surveillance 


\section{INTRODUÇÃO}

No Brasil, o marco regulatório para registro de medicamentos e outros produtos sujeitos à vigilância sanitária é a Lei Federal $\mathrm{n}^{\circ} 6.360$, de 23 de setembro de $1976^{1}$, que dispõe sobre medicamentos, drogas, insumos farmacêuticos e correlatos, cosméti$\cos$, saneantes e outros produtos. Essa lei determina que nenhum desses produtos, inclusive os importados, poderá ser industrializado, exposto à venda ou entregue ao consumo antes de registrado. Além disso, principalmente nos casos dos medicamentos, devem ser comprovadas a eficácia e a segurança por meio de ensaios clínicos, devidamente estruturados e aprovados ${ }^{1}$.

A Agência Nacional de Vigilância Sanitária (Anvisa) define ensaio clínico como:

pesquisa conduzida em seres humanos com o objetivo de descobrir ou confirmar os efeitos clínicos e/ou farmacológicose/ou qualquer outro efeito farmacodinâmico do medicamento experimental e/ou identificar qualquer reação adversa ao medicamento experimental e/ou estudar a absorção, distribuição, metabolismo e excreção do medicamento experimental para verificar sua segurança e/ou eficácia².

0 arcabouço legislativo brasileiro a respeito de pesquisa clínica divide-se em duas principais vertentes: ética e técnica. As informações éticas foram as primeiras que surgiram no cenário brasileiro, tendo em vista todo o cenário mundial que se construiu após a segunda guerra. Dessa forma, era necessário que o Brasil tivesse suas próprias regras éticas para evitar que sua população ficasse exposta a possíveis danos recorrentes da pesquisa clínica. A primeira vez que a figura do comitê de ética foi citada na legislação brasileira ocorreu em 1988, quando o Conselho Nacional de Saúde publicou a Resolução ${ }^{\circ}{ }^{\circ}$, de 13 de junho ${ }^{3}$. A Resolução $\mathrm{n}^{\circ}$ 196, de 10 de outubro de 1996, também de natureza bioética, revogou a anterior e aprovou diretrizes e normas regulamentadoras de pesquisas envolvendo seres humanos. Essa resolução trouxe termos e definições relacionados à pesquisa clínica e citou, pela primeira vez, o Termo de Consentimento Livre e Esclarecido (TCLE); estabeleceu a composição, as competências e a formação da Comissão Nacional de Ética em Pesquisa (Conep) e dos Comitês de Ética em Pesquisa (CEP); os protocolos de pesquisa e as considerações sobre o risco e o benefício em ensaios clínicos ${ }^{4}$. Em 2012, essa Resolução foi substituída pela ${ }^{\circ}$ 466 de 12 de dezembro ${ }^{5}$, que é sobre bioética e está atualmente vigente no Brasil.

Nesse cenário ético, surgem duas instâncias fundamentais: CEP e Conep. As instituições de pesquisa podem ter seu próprio CEP, desde que sigam as regras da Conep. Os CEP avaliam os projetos de pesquisa e, em alguns casos, esses protocolos seguem para análise complementar da Conep. 0 objetivo principal dessa análise é proteger o participante da pesquisa, a equipe, a instituição, a sociedade e o meio ambiente ${ }^{6}$.

A Conep é uma instância colegiada de natureza consultiva, deliberativa, normativa e educativa ${ }^{7}$. Os projetos das seguintes áreas temáticas necessitam da avaliação da Conep: genética humana, reprodução humana, equipamentos ou dispositivos terapêuticos novos ou não registrados no Brasil, novos procedimentos terapêuticos invasivos, estudo com população indígena, organismos geneticamente modificados ou células-tronco ou organismos que representem alto risco coletivo e coordenação e/ou patrocínio de fora do país, exceto os de copatrocínio do governo brasileiro. Também são encaminhados para a Conep aqueles protocolos que os CEP julgarem necessários, cuja instituição proponente seja o Ministério da Saúde ou projetos que não tenham instituição proponente (Conep escolhe um CEP para avaliar) ${ }^{5}$.

Para encaminhar o projeto de pesquisa para o sistema CEP/Conep é necessário cadastrar os documentos por meio da Plataforma Brasil, que é um sistema computadorizado que integra todos esses participantes de análise ética. Essa submissão via plataforma Brasil pode ser paralela com a submissão do ensaio clínico para a Anvisa.

A Anvisa é a responsável, desde sua criação, em 1999, pelos registros de tecnologias em saúde no Brasil e também pelas regras para análise das questões técnicas de ensaios clínicos. A primeira Resolução da Diretoria Colegiada (RDC) que versava sobre esse tema foi a RDC $n^{\circ} 219$, de 20 de setembro de $2004^{8}$, que apresentava o regulamento para elaboração do dossiê de pesquisa clínica para a obtenção de um comunicado especial (CE), que é o documento necessário para a importação de produtos com a finalidade de serem utilizados na pesquisa clínica.

A RDC $n^{\circ} 39$, de 5 de junho de $2008^{9}$ revogou a RDC $n^{\circ}$ 219/2004. Essa normativa, que dispunha das regras para aprovar a realização de pesquisas clínicas no Brasil, trazia informações sobre o tipo de documentação que deveria ser apresentada à Agência, as regras para elaboração de dossiês de pesquisa clínica e a obtenção do CE.

Essa Resolução foi revogada pela atual regra de pesquisa clínica com medicamentos no Brasil, elaborada pela Anvisa, a $\operatorname{RDC~} n^{\circ} 9$, de 20 de fevereiro de $2015^{2}$.

A RDC $n^{\circ} 9 / 2015^{2}$ atualizou o arcabouço regulatório a respeito de ensaios clínicos com medicamentos no país, com o objetivo de reduzir o tempo de análise dos pleitos por ensaios clínicos e ter uma abordagem mais parecida com o que é aplicado por outras agências regulatórias, visando atrair ensaios clínicos para o país ${ }^{10}$. Essa legislação tem como escopo os ensaios clínicos com medicamentos que terão todo ou parte de seu desenvolvimento clínico no Brasil para fins de registro. Além disso, os ensaios clínicos com medicamentos registrados no Brasil devem seguir todas as disposições desta Resolução quando fornecerem subsídios para: nova indicação terapêutica, nova via de administração, nova concentração, nova forma farmacêutica, ampliação de uso, nova posologia, novas associações ou qualquer alteração pós-registro que requeira dados clínicos, incluindo renovação de registro.

Atrair mais estudos para o Brasil permite que existam dados disponíveis sobre eficácia e segurança de medicamentos específicos 
para essa população ${ }^{3}$. Embora tenha adotado essa medida, a Anvisa em suas normas que regulamentam o registro de medicamentos, possibilita a apresentação de estudos clínicos realizados em outros países, conforme norma específica ${ }^{11,12,13,14,15}$. A validade externa do ensaio clínico, que é a capacidade de generalização dos dados para a população geral, pode ficar comprometida, considerando as características da população estudada, tais como diferenças farmacogenômicas, diferentes doenças-base, perfil farmacoepidemiológico e esquemas nutricionais ${ }^{16}$.

Como exemplo, dessas diferenças farmacogenômicas, pode-se citar o fato de que os afroamericanos têm maior hepatotoxicidade quando expostos ao paracetamol; caucasianos, menor toxicidade hepática no uso de isoniazida, maior toxidade com o uso de varfarina e maior risco de hipoglicemia com uso de glibenclamida; judeus ocidentais têm prolongamento de paralisia muscular com suxametônio ${ }^{17}$. Além disso, a eficácia clínica relacionada ao uso de anti-hipertensivos e a resposta inicial a anticoagulantes orais foram menores entre a população negra ${ }^{18,19}$.

As respostas aos medicamentos também podem ser afetadas por fatores extrínsecos, como: clima, poluição, cultura, práticas médicas e uso de medicamentos que diferem entre países. De acordo com o guia E5 - Ethnic Factors In The Acceptability of Foreign Clinical Data, do International Conference on Harmonisation $(\mathrm{ICH})$, organização que trata sobre harmonização de práticas regulatórias entre os países signatários, os fatores intrínsecos precisam ser avaliados entre as populações, pois, a partir deles, é que será possível ou não determinar generalizações dos estudos clínicos, enquanto que os extrínsecos afetam o desenho e a conduta na pesquisa clínica ${ }^{20}$. Outro guia relevante, também emitido pela ICH, o qual o Brasil é signatário, é o E17 - General Principles For Planning and Design of Multi-Regional Clinical Trials. Esse documento traz as recomendações básicas que precisam ser seguidas para a condução de ensaios clínicos multirregionais, ou seja, em diferentes países ${ }^{21}$.

Outro ponto relevante, nesse contexto, é a migração atual da realização de ensaios clínicos de países desenvolvidos para países de renda baixa e média, com economias emergentes. Drain et al. ${ }^{22}$ publicaram um estudo em 2018, apresentando os 20 países que tiveram as maiores taxas de crescimento no número de ensaios clínicos, realizados localmente, entre 2006 e 2012. O Brasil não se encontra nesse ranking, mas países da América Latina, como Colômbia, Venezuela e Panamá, estão listados. Essa falta de crescimento expressivo no Brasil, quando comparado com outros países latino-americanos, precisa ser discutida e os motivos levantados.

Neste sentido, incluir no arcabouço da legislação sanitária brasileira medidas para atrair o interesse para a realização de ensaios clínicos com nossa população favorece a tomada de decisão para o registro de produtos e colabora para a elaboração do plano de gestão de riscos associados. Esse plano elenca os principais riscos e pontos críticos da pesquisa e apresenta sugestões para a sua minimização.

Todas essas atualizações normativas, éticas e técnicas refletem um esforço brasileiro para que o país se torne mais participativo no cenário mundial de realização de pesquisas clínicas. A publicação da RDC $n^{\circ} 9 / 2015^{2}$ foi um marco legal importante e, por isso, marca o desenvolvimento do presente estudo, que teve como objetivo caracterizar os ensaios clínicos de fase 3 realizados no Brasil a partir da publicação da referida norma, avaliando esse progresso no país na perspectiva mundial.

\section{MÉTODO}

Trata-se de estudo exploratório e descritivo, realizado em três etapas. Na primeira, a fim de identificar mudanças no número de ensaios clínicos com medicamentos realizados no Brasil a partir da publicação da Resolução RDC $n^{\circ} 9 / 2015^{2}$, realizou-se análise quantitativa comparando dois momentos: antes e depois da vigência da atual norma que trata sobre pesquisa clínica com medicamentos no Brasil. A fim de determinar o período de avaliação, calculou-se o número de dias entre o início de vigência da norma (3 de março de 2015) até a data de fechamento dessa pesquisa (25 de novembro de 2019), ou seja, 1.728 dias. Desta forma, a fim de avaliar iguais períodos em dias, a avaliação pré-resolução foi compreendida entre 9 de junho de 2010 e 2 de março de 2015. Além do período de avaliação, foram utilizados os seguintes filtros na busca: Interventional Studies | Brazil | Phase 3.

Na segunda etapa, realizou-se análise de todos os registros deferidos pela Anvisa e que tinham Parecer Público de Avaliação do Medicamento (PPAM) desde a instauração do banco de dados em 2015 até a data de finalização da primeira fase deste estudo (25 de novembro de 2019), com a finalidade de verificar se as aprovações estavam amparadas em estudos realizados com população brasileira. A escolha da análise dos PPAM para esse trabalho foi realizada, uma vez que esse documento apresenta informações detalhadas sobre o registro do medicamento, o que possibilita a verificação se o registro foi com base em estudos internacionais, por exemplo. Além disso, a data de implementação do PPAM foi contemporânea com a da RDC $n^{\circ} 09 / 2015^{2}$, alvo desse estudo.

Na terceira e última etapa, realizou-se análise completa das características dos ensaios clínicos que foram realizados após a vigência da RDC $n^{\circ} 9 / 2015^{2}$, registrados na base de dados internacional Clinicaltrials.gov e no banco de dados brasileiro de ensaios clínicos, Registro Brasileiro de Ensaios Clínicos (ReBEC).

A base de dados Clinicaltrials.gov foi escolhida para essa análise, pois ela está entre os maiores repositórios de estudo clínicos e possui campos de pesquisa bastante abrangentes. Embora esse não seja o banco de dados mais abrangente de ensaios clínicos (em comparação com Plataforma Internacional de Registro de Ensaios Clínicos - ICTRP), é uma base bem completa e de fácil pesquisa ${ }^{23}$.

Os estudos encontrados em ambas as bases foram considerados apenas uma vez para esse estudo. Foram aplicados, para tanto, os filtros: Recruiting, Active, not recruiting, Completed, Enrolling by invitation, Suspended, Terminated, Withdrawn Studies | Interventional Studies | Brazil | Phase 3 | Start date from 03/03/2015 to 11/25/2019. Dessa forma, ao aplicar os filtros anteriormente citados, apenas estudos com braço no Brasil, fase III, no período de tempo definido e com as situações 
descritas foram encontrados na plataforma. Não foram aplicados os filtros de "recrutamento não iniciado", pois não era possível saber se o recrutamento seria autorizado e "estado indefinido", uma vez que estudos sem estado definido poderiam causar confusão em relação aos dados encontrados. Apenas estudos fase III foram incluídos, pois, normalmente, eles são a fase final de análise clínica obrigatória, antes do registro ${ }^{11,12,13,14,15}$. Como o objetivo é fazer uma avaliação da situação dos registros associados aos ensaios clínicos, o foco foi direcionado para essa fase. Desses estudos, foram avaliadas suas principais características como: intervenção, comparador, classificação da intervenção (sintético, biológico, específico, fitoterápico, entre outros), patrocinador e tipo do patrocinador (indústria farmacêutica, filantropia, pesquisa por meio de universidades e agências de fomento). Para a classificação do patrocinador, foi realizada busca no site da instituição citada como patrocinadora.

A base de cálculo para essas análises foi o uso de proporções numéricas. O universo amostral era considerado como $100 \%$ e o somatório para cada uma das análises foi calculada em relação ao total.

Como o estudo utiliza dados de acesso público e sem identificação de participantes, não foi necessária a submissão do projeto do estudo à apreciação de Comitê de Ética em Pesquisa em Seres Humanos.

\section{RESULTADOS E DISCUSSÃO}

A RDC n 9/20155, marco regulatório objeto deste estudo, desburocratizou alguns aspectos da análise de pesquisas clínicas, tais como a diminuição do tempo de análise do protocolo do estudo para 90 dias, salvo exceções; análise paralela com órgãos reguladores éticos, novo formato de documento a ser apresentado para a Anvisa - o Dossiê de Desenvolvimento Clínico de Medicamento (DDCM), que se assemelha aos formatos utilizados por outros países.

Na primeira etapa do estudo, foi observado que o total de ensaios clínicos de fase III realizados no Brasil no período pré-RDC foi de 653 ensaios clínicos e, no período pós-vigência, de 525, representando uma redução de $20 \%$, o que sugere que apenas a publicação da norma não foi suficiente para atrair pesquisas para o país. 0 desenvolvimento clínico de um medicamento se divide em quatro fases, principalmente. Na fase I, o medicamento é testado em voluntários saudáveis para avaliação de sua segurança. A fase II é um estudo piloto de eficácia e segurança, já com a população-alvo do tratamento. A fase III é um estudo com um número bem maior de participantes e é considerada como o padrão-ouro dos estudos intervencionais. A fase IV abrange a análise do medicamento, após o registro, na vida real ${ }^{24}$.

O Brasil apresenta pontos positivos e negativos para a realização de ensaios clínicos localmente. Como principal ponto forte, existe a facilidade de recrutamento e como ponto negativo a demora regulatória para aprovação dos estudos. Essa burocracia afeta a realização de estudos no país, incentivando a estratégia de registro de produtos sem a realização de pesquisa clínica no Brasil25. Assim, mesmo com mudança no arcabouço legislativo, o Brasil ainda enfrenta dificuldades em se inserir como polo de pesquisa clínica mundial, demonstrando que a nova legislação ainda não teve os impactos esperados no cenário brasileiro.

Existem diversas implicações para a realização de ensaios clínicos em países em desenvolvimento em detrimento de países desenvolvidos. Entre elas, pode-se citar: que a população com um menor nível econômico e educacional acaba sendo mais suscetível ao aceite na participação desses ensaios; a falta de assistência médica no local gerando expectativa de tratamento para a população; a possibilidade de captação e amostras maiores para o estudo; a oportunidade de novo mercado para a indústria farmacêutica ${ }^{26}$. Entretanto, os resultados sugerem que ainda há outros entraves para o Brasil ser incluído na rota preferencial de realização de ensaios clínicos pelos patrocinadores.

A ausência de um guia brasileiro de Boas Práticas Clínicas, por exemplo, foi uma lacuna na legislação brasileira até 2008, quando a Anvisa incorporou o Documento das Américas de Boas Práticas Clínicas'. E, em novembro de 2019, a Agência traduziu para o português "Guia Harmonizado do $\mathrm{ICH}$ - Adendo integrado ao ICH e6(r1): Guia de Boas Práticas Clínicas E6(r2)”27, definido como um padrão internacional de ética e qualidade científica para desenhar, conduzir, registrar e reportar ensaios que envolvam a participação de seres humanos. Segundo a publicação, o cumprimento desse padrão oferece uma garantia pública de que os direitos, a segurança e o bem-estar dos participantes do ensaio estão protegidos, de forma consistente com os princípios que têm sua origem na Declaração de Helsinque, e que os dados do ensaio clínico têm credibilidade.

Shenoy ${ }^{28}$ avaliou as regras globais para estudos multicêntricos e seus achados reforçaram a definição de requisitos mínimos de qualidade mundiais para que um estudo clínico possa ser realizado. Esses requisitos mínimos incluíam: análise de diferenças intrínsecas e extrínsecas, análise de diferenças de terapias base, harmonização das boas práticas clínicas, randomizações considerando a variabilidade populacional, entre outros. A definição desses critérios de qualidade pode ajudar não só em uma realização multicêntrica de pesquisa clínica mais harmônica, tornando mais previsível e menos oneroso todo o processo e, por consequência, atrair mais estudos para o Brasil, mas, por outro lado, tende a harmonizar o processo entre países, incentivando que estudos clínicos realizados em diferentes nações sirvam de base para o registro de medicamentos em outras localidades, devido à inviabilidade financeira e temporal de se realizar os estudos em todos os países de interesse comercial29.

Pelo exposto, a segunda etapa deste estudo analisou os registros de medicamentos no Brasil no período pós-RDC $n^{\circ}$ 9/20152, publicados via PPAM. Dos 295 registros encontrados no período de busca, 115 eram genéricos, 86 biológicos, 74 novos, seis similares, 13 específicos e um fitoterápico. É importante citar que os medicamentos classificados como genéricos e similares $(n=121)$ são isentos de pesquisa clínica para o registro, uma vez que sua segurança e eficácia são comprovadas por meio de estudos de bioequivalência ${ }^{15}$. Além disso, medicamentos específicos e fitoterápicos também podem ter sua segurança e eficácia comprovadas por outros meios como tradicionalidade de uso ou literatura 
científica ${ }^{12,13}$. Neste estudo, foi verificado que três medicamentos da classe de medicamentos específicos e o fitoterápico (um) foram registrados sem condução de pesquisas clínicas e, para três produtos biológicos, a informação não estava disponível.

Desta forma, foram avaliadas as populações que embasaram o registro de 167 medicamentos restantes, sendo que apenas 56 (34\%) deles foram registrados no Brasil com base em pesquisas clínicas realizadas com brasileiros (Figura 1). Para dois medicamentos a informação não estava disponível.

Este resultado pode estar relacionado com a baixa atratividade do Brasil para sediar ensaios clínicos, conforme verificado na primeira etapa, uma vez que a maior parte dos medicamentos registrados no Brasil apresenta estudos clínicos realizados fora no país. Se o Brasil fosse um país atrativo para a realização de ensaios clínicos, seria esperado que a maior parte dos medicamentos registrados no país estivesse embasada em estudos na população brasileira, visto que a chance de o registro não ser aceito no país, por problemas de farmacogenômica, seria eliminada. Alvarenga e Martins ${ }^{30}$ verificaram que os países emergentes eram o principal destino para ensaios clínicos. Adobor ${ }^{31}$ reforçou esse achado, afirmando que países emergentes, com seus mercados também emergentes, são considerados como referência para o recebimento de novos ensaios clínicos. Foi observado que não existe uma preferência específica por alguns países como sede de estudos e que o Brasil poderia ser incluído em estudos multicêntricos, pela facilidade de recrutamento.

Em um estudo realizado pelo Banco Nacional de Desenvolvimento Econômico e Social (BNDES), foram apresentadas informações sobre os principais desafios e atrativos dos estudos clínicos no Brasil. Nesse trabalho, foi abordada a questão de registro de medicamentos com ensaios clínicos internacionais em outros países e no Brasil. No país, essa prática é comum e não existe uma recomendação específica de que os estudos precisam ser realizados em uma parcela da população brasileira, conforme é recomendado em outros países ${ }^{25}$, o que pode explicar os achados desta etapa do presente estudo.

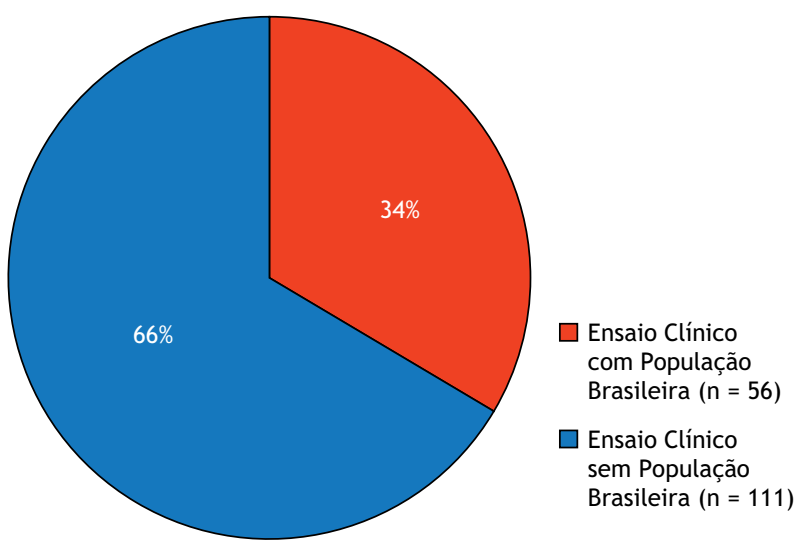

Fonte: Elaborada pelos autores com base nos dados coletados via Parecer Público de Avaliação do Medicamento (PPAM), 2020.

Figura 1. Comparação dos registros de medicamentos no Brasil quanto à realização de ensaios clínicos no Brasil $(n=167)$.
Relatório de pesquisa de mercado publicado pela Associação da Indústria Farmacêutica de Pesquisa (Interfarma), intitulado “A Importância da Pesquisa Clínica para o Brasil”31, afirmou que o Brasil figura na $24^{a}$ colocação no ranking mundial de pesquisa clínica, com apenas 2,1\% dos estudos. Segundo os autores, esse dado representa uma queda de sete posições em dez anos. Afirmaram, ainda, que o melhor aproveitamento do potencial brasileiro, poderia saltar para a $10^{\text {a }}$ colocação, atraindo um investimento estimado de $\mathrm{R} \$ 2$ bilhões, com efeitos na economia de cerca de $\mathrm{R} \$ 5$ bilhões. Essa queda no ranking mundial é sinônimo de baixo investimento do governo em desenvolvimento de tecnologias e reduzido número de contratações de pessoal qualificado para os órgãos reguladores. Além disso, a falta de harmonização nas exigências éticas continua sendo um entrave ${ }^{32}$.

Realizar estudos clínicos em países diferentes daqueles que se pretende registrar o produto é uma discussão muito ampla. Gor$\mathrm{ski}^{33}$ afirmou que muitos pacientes são prejudicados diariamente por não possuírem no mercado de seus países um medicamento novo, eficaz e seguro, já disponível em outras localidades, para suas enfermidades. Uma das razões para isso é que as agências reguladoras que se deparam com estudos clínicos realizados em outros países tendem a ser mais rigorosas e, algumas vezes, até demandam novos estudos locais. Contudo, o não aceite arbitrário desses estudos é criticado, uma vez que isso acaba desabastecendo o mercado e é considerada uma ótima oportunidade de aumentar a comunicação entre as diferentes agências regulatórias do mundo.

$\mathrm{Na}$ União Europeia, a European Medicines Agency (EMA) publicou, em 2009, uma reflexão sobre a extrapolação de dados clínicos internacionais para a população europeia. Nesse documento foi informado que estudos internacionais podem ser aceitos no pedido de registro, contudo, é necessária uma análise caso a caso com a finalidade de avaliar se o estudo abrange características intrínsecas e extrínsecas que possam corresponder aos europeus $^{34}$. Na tentativa de melhorar essas análises dos estudos feitos em outros países, a agência sanitária norte-americana, o Food and Drug Administration (FDA), tem implementado escritórios em outros países e aumentado as inspeções internacionais ${ }^{35}$. A alta variabilidade entre as populações gera inseguranças nas agências reguladoras. Agências reguladoras internacionais, como as da Coreia do Sul, Índia e Taiwan, solicitam que o medicamento tenha sido testado em pelo menos uma parcela de sua população antes que ele seja registrado no país ${ }^{25}$.

O Brasil apresenta uma situação muito específica, pois sua população é considerada tri-híbrida, ou seja, descendente de africanos, europeus e índios americanos. Nesse sentido, selecionar uma parcela de determinada etnia pode não ser representativo, geneticamente, à população como um todo, o que torna necessários mecanismos adicionais de acompanhamento do uso do medicamento no país ${ }^{36}$. Estudos vêm sendo feitos para aplicar programas de análise genômica associada à estatística aplicada para avaliar a heterogeneidade da população brasileira e suas possíveis implicações nas decisões que tangem o controle regulatório ${ }^{37}$. 
Ademais, existe uma diferença entre genética e etnicidade. A genética está relacionada com características físicas das pessoas, como: cor da pele, caminhos metabólicos, aspectos faciais, entre outros, e a etnicidade refere-se mais aos aspectos culturais como: religião, nível educacional e dieta. Dessa forma, na pesquisa clínica, a genética influencia aspectos farmacogenômicos de resposta a medicamentos, enquanto a etnicidade influencia na participação ou não no estudo. Diante disso, fica clara a necessidade de inclusão de população de vários países em estudos que visam o registro de novos medicamentos no mundo ${ }^{38}$.

Para identificar as principais características dos ensaios clínicos realizados no Brasil após a vigência da RDC $n^{\circ} 9 / 2015^{2}$, foi realizada uma triagem completa referente a esses estudos (etapa 3 ). Entre a vigência da referida RDC até a data de fechamento dessa pesquisa, foram encontrados 525 estudos conduzidos no Brasil, dos quais 33 foram excluídos: três por serem duplicados, 29 não tinham como intervenção medicamento e um era estudo de fase 2 . Desta forma, foram analisadas as características de 492 estudos.

Em relação às classes de medicamentos utilizados nesses ensaios clínicos, foi possível verificar que as classes mais estudadas eram a de sintéticos $(n=269 ; 55 \%)$ e de biológicos $(n=203 ; 41 \%)$, totalizando 479 estudos dos 492 avaliados (97\%) (Figura 2).

Os resultados expostos na Figura 2 são esperados, tendo em vista que a Anvisa exige comprovação de eficácia e segurança, de forma insubstituível, para os medicamentos enquadrados nas categorias de medicamentos sintéticos (novos/inovadores) e biológicos. Ademais, as áreas para as quais há mais investimento em pesquisa clínica no mundo são oncologia e sistema nervoso central, as quais possuem grande quantidade de biológicos e sintéticos, respectivamente, além de estar em plena ascensão as terapias genéticas ${ }^{32}$.

Outra característica analisada relacionada aos estudos clínicos realizados no Brasil foi o comparador escolhido para o desenho: se as terapias estudadas eram comparadas com o placebo ou com outro medicamento indicado para a mesma enfermidade.

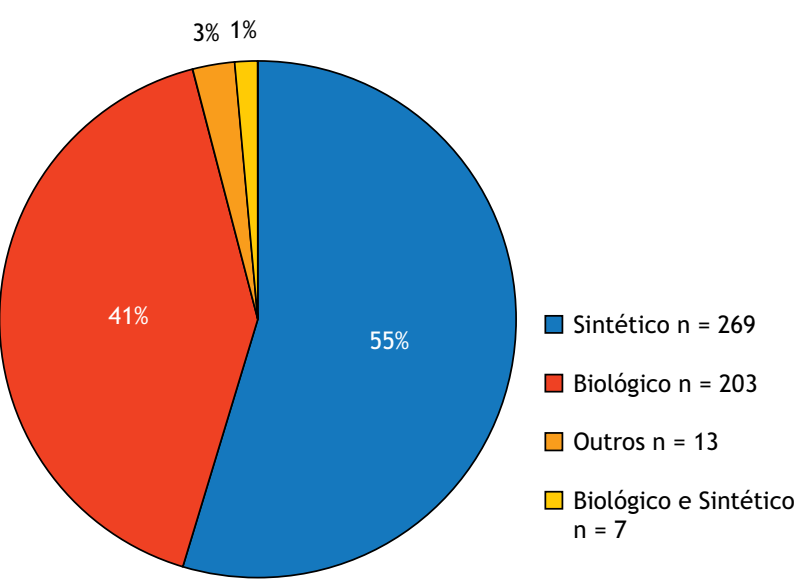

Fonte: Elaborada pelos autores com base nos dados de clinicaltrials.com, 2020.

Figura 2. Comparação dos ensaios clínicos realizados no Brasil por classificação de medicamento $(n=492)$.
Foi possível perceber que ainda existe um grande uso exclusivo de placebo como comparador $(n=182 ; 37 \%$ ) (Figura 3), fato que deve ser avaliado com cautela, principalmente, focando na justificativa de uso exclusivo de placebo no estudo.

Por fim, deve haver extremo rigor na análise ética para aprovação de desenhos de ensaios clínicos que utilizam como comparador o placebo, a fim de proteger os direitos, a segurança e o bem-estar dos participantes da pesquisa. No Brasil, essa análise é de competência do sistema CEP/Conep, tendo como norma base a Resolução $n^{\circ} 466 / 2012$ do Conselho Nacional de Saúde (CNS) ${ }^{39}$.

O tipo de financiamento de ensaios clínicos também é um dado importante a ser analisado, uma vez que os estudos podem ser financiados pelas indústrias farmacêuticas que fabricam os medicamentos ou por instituições de pesquisa e fomento, sem os devidos fins lucrativos. Considerando a situação brasileira, foi possível identificar que a grande maioria dos estudos é patrocinada pelas indústrias farmacêuticas $(n=431 ; 88 \%)$ (Figura 4).

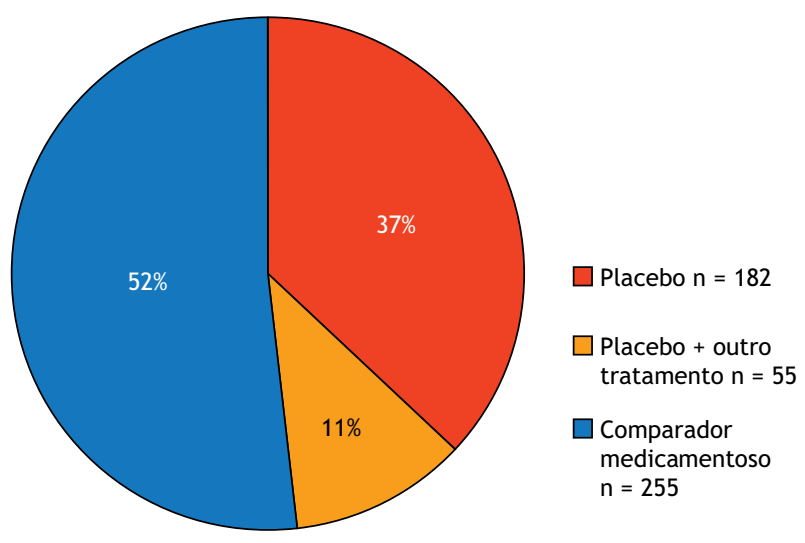

Fonte: Elaborada pelos autores com base nos dados de clinicaltrials.com, 2020.

Figura 3. Comparação dos ensaios clínicos realizados no Brasil em relação ao comparador escolhido $(n=492)$.

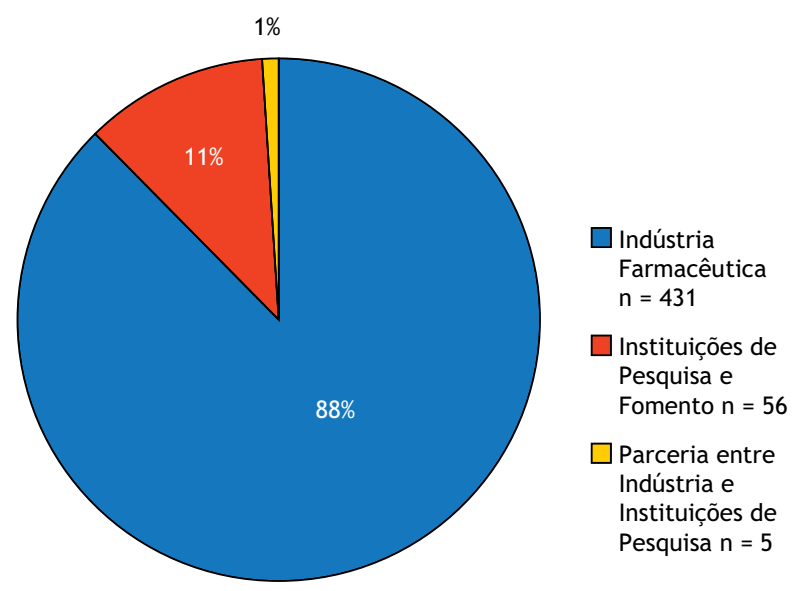

Fonte: Elaborada pelos autores com base nos dados de clinicaltrials.com, 2020.

Figura 4. Comparação dos ensaios clínicos realizados no Brasil em relação ao financiador $(n=492)$. 
Diante da análise das demais características dos estudos realizados no Brasil, foi possível observar que a indústria farmacêutica é o maior patrocinador dos estudos. Estudo brasileiro ${ }^{40}$ corrobora com esses achados. Os autores concluíram que, em geral, os ensaios clínicos conduzidos no Brasil estão marcados pela grande influência mercadológica, com financiamento privado e com projetos que são apenas extensões de pesquisas originadas em outros países.

Diante da análise das três etapas deste estudo, verificou-se que um expressivo número de medicamentos tem sido registrado no Brasil ainda com estudos em população estrangeira, utilizando o placebo como comparador e, como principal patrocinador dos estudos, a indústria farmacêutica, nos resultados apurados, o que torna imprescindíveis a priorização e o fortalecimento de ações de farmocovigilância no país, a fim de garantir que a monitorização do uso em vida real dos produtos registrados gere dados de segurança e eficácia aplicados à população brasileira, baseando análise em seus fatores intrínsecos e extrínsecos.

Essa aparenta ser a melhor solução porque não parece interessante que sejam aplicadas regras para exigência de estudos clínicos nacionais para registro de produtos farmacêuticos no Brasil, pois, provavelmente, tornaria o mercado brasileiro ainda menos atrativo para realização de ensaios, e, por sua vez, registro de produtos, uma vez que a escolha do local pela indústria farmacêutica ou empresa interessada no ensaio clínico, está relacionada a um país que tenha como características importantes a velocidade de recrutamento, melhor infraestrutura, menores custos, maior capacitação de pessoal e ambiente ético-regulatório adequados ${ }^{25}$.

Os estudos clínicos continuam sendo concentrados em países desenvolvidos. Isso acontece pela alta capacitação e maior quantitativo de pessoal capacitado e contratado para atuar no cenário da pesquisa clínica, que demanda grande envolvimento intelectual ${ }^{41}$. Dessa forma, alterar o arcabouço regulatório, trazendo facilidades de prazos e harmonização com entes internacionais, se faz necessário, mas pode não ser suficiente. É preciso que um maior investimento seja feito nessa área, com o objetivo de criar uma boa base de conhecimento e mão de obra qualificada, que culminará em uma melhoria geral desse cenário brasileiro.

Se faz importante salientar que esse trabalho apresenta como limitação a não utilização da base de dados ICTRP, devido à inviabilidade de buscas nessa base e, com isso, alguns estudos realizados no Brasil podem ter sido desconsiderados na pesquisa, pela utilização apenas do ClinicalTrials.gov e Rebec.

Como esse trabalho focou em pesquisa relacionada apenas a datas, nenhum termo Medical Subject Headings (Mesh) ou Descritores em Ciências da Saúde (Decs) foi utilizado, uma vez que todos os estudos encontrados na busca por data foram considerados para a análise.

Os ensaios clínicos incluídos no presente artigo não foram categorizados segundo à Classificação Estatística Internacional de Doenças e Problemas Relacionados à Saúde (CID). Isso se deve ao fato de que a classificação dos tratamentos foi realizada com o foco no enquadramento regulatório do medicamento estudado. Foi dada preferência a esse tipo de classificação, considerando o foco regulatório desse trabalho.

\section{CONCLUSÕES}

Esse estudo verificou que a tentativa de tornar a regulação para ensaios clínicos mais atraentes no Brasil parece não ter sido suficiente, uma vez que houve redução de ensaios clínicos, quando se compara o período anterior e posterior da publicação da RDC $n^{\circ} 9 / 2015^{2}$ da Anvisa. Dentre os ensaios clínicos registrados e realizados, placebo ainda é muito utilizado como comparador e a população brasileira está presente na minoria dos estudos. Por fim, diante deste cenário, é imperativo que se fortaleçam as ações de farmacovigilância no Brasil, a fim de conhecer os perfis de efetividade e segurança dos medicamentos após exposição da população brasileira.

\section{REFERÊNCIAS}

1. Brasil. Lei $\mathrm{N}^{\circ} 6.360$, de 23 de setembro de 1976. Dispõe sobre a vigilância sanitária a que ficam sujeitos os medicamentos, as drogas, os insumos farmacêuticos e correlatos, cosméticos, saneantes e outros produtos, e dá outras providências. Diário Oficial União. 24 set 1976.

2. Agência Nacional de Vigilância Sanitária - Anvisa. Resolução RDC № 9, de 20 de fevereiro de 2015. Dispõe sobre o regulamento para a realização de ensaios clínicos com medicamentos no Brasil. Diário Oficial União. 3 mar 2015.

3. Conselho Nacional de Saúde - CNS. Resolução $N^{\circ} 1$, de 10 de outubro de 1988. Aprova as normas de pesquisa em saúde. Diário Oficial União. 11 out 1988.

4. Conselho Nacional de Saúde - CNS. Resolução Nº 196, de 10 de outubro de 1996. Diretrizes e normas regulamentadoras de pesquisa envolvendo seres humanos. Diário Oficial União. 11 out 1996.
5. Conselho Nacional de Saúde - CNS. Resolução N 466 , de 12 de dezembro de 2012. Aprova as seguintes diretrizes e normas regulamentadoras de pesquisas envolvendo seres humanos. Diário Oficial União. 13 jun 2013.

6. Alvarenga L. Princípios e diretrizes das boas práticas clínicas: GCP, ICH e documento das Américas em II curso de capacitação em pesquisa clínica. São Paulo: Hospital Alemão Oswaldo Cruz; 2011.

7. Werner JAT, Velho MTC. O sistema CEP/Conep no Brasil: discurso e realidade. Rev Direito. 2008;(29):166-80. https://doi.org/10.17058/rdunisc.v0i29.565

8. Agência Nacional de Vigilância Sanitária - Anvisa. Resolução RDC No 219, de 20 de setembro de 2004. Aprova o regulamento para elaboração de dossiê para a obtenção de comunicado especial (CE) para realização de pesquisa clínica com medicamentos e produtos para a saúde. Diário Oficial União. 21 set 2004. 
9. Agência Nacional de Vigilância Sanitária - Anvisa. Resolução $\mathrm{RDC} \mathrm{N}^{\circ} 39$, de 5 de junho de 2008. Aprova o regulamento para a realização de pesquisa clínica e dá outras providências. Diário Oficial União. 1 ago 2008.

10. Fagundes P, Dresel P, Miler AE. Brazil's regulatory environment offers positive changes for clinical trials. Regulatory Focus. 2018.

11. Agência Nacional de Vigilância Sanitária - Anvisa. Resolução RDC N ${ }^{\circ} 55$, de 10 de dezembro de 2010. Dispõe sobre o registro de produtos biológicos novos e produtos biológicos e dá outras providências. Diário Oficial União. 17 dez 2010.

12. Agência Nacional de Vigilância Sanitária - Anvisa. Resolução RDC No 24, de 14 de junho de 2011. Dispõe sobre o registro de medicamentos específicos. Diário Oficial União. 17 jun 2011.

13. Agência Nacional de Vigilância Sanitária - Anvisa. Resolução $\mathrm{RDC} \mathrm{N}^{\circ} 26$, de 13 de maio de 2014. Dispõe sobre o registro de medicamentos fitoterápicos e o registro e a notificação de produtos tradicionais fitoterápicos. Diário Oficial União. 14 maio 2014

14. Agência Nacional de Vigilância Sanitária - Anvisa. Resolução RDC Nº 60, de 10 de outubro de 2014. Dispõe sobre os critérios para a concessão e renovação do registro de medicamentos com princípios ativos sintéticos e semissintéticos, classificados como novos, genéricos e similares, e dá outras providências. Diário Oficial União. 14 out 2014.

15. Agência Nacional de Vigilância Sanitária - Anvisa. Resolução RDC № 200, de 26 de dezembro de 2017. Dispõe sobre os critérios para a concessão e renovação do registro de medicamentos com princípios ativos sintéticos e semissintéticos, classificados como novos, genéricos e similares, e dá outras providências. Diário Oficial União. 29 jan 2018.

16. Rebelo Neto MJ. Farmacogenética/farmacogenómica [dissertação]. Porto: Universidade Fernando Pessoa; 2013.

17. Weber WW. The legacy of pharmacogenetics and potential applications. Mutat Res. 2001;479(1-2):1-18. https://doi.org/10.1016/S0027-5107(01)00151-8

18. Nadruz W, Claggett B, Rosamond WD, Folsom AR, Solomon SD. Racial disparities in risks of stroke. N Engl J Med. 2017;376(21):2089-90. https://doi.org/10.1056/NEJMc1616085

19. Essien UR, Magnani JW, Chen N, Gellad WF, Fine MF, Hernandez I. Race/ethnicity and sex-related differences in direct oral anticoagulant initiation in newly diagnosed atrial fibrillation: a retrospective study of medicare data. J Natl Med Assoc. 2020;112(1):103-8. https://doi.org/10.1016/j.jnma.2019.10.003

20. European Medicines Agency - EMA. Ethnic factors in the acceptability of foreign clinical data. Amsterdam: European Medicines Agency; 1998.

21. European Medicines Agency - EMA. ICH guideline E17 on general principles for planning and design of multi-regional clinical trials. Amsterdam: European Medicines Agency; 2017.

22. Drain PK, Parker RA, Robine M, Holmes KK. Global migration of clinical research during the era of trial registration. PLoS One. 2018;13(2):1-13. https://doi.org/10.1371/journal.pone. 0192413

23. Jaffe IS, Chiswell K, Tsalik EL. A decade on: systematic review of clinicaltrials gov infectious disease trials, 2007-2017. Open Forum Infect Dis. 2019;6(6):1-9. https://doi.org/10.1093/ofid/ofz189

24. Barboza MFS. Manual básico para realização de ensaios clínicos conduzidos por um investigador-patrocinador: abordagem dos procedimentos por gestão de processos [dissertação]. Rio de Janeiro: Fundação Oswaldo Cruz; 2015.

25. Gomes RP, Pimentel VP, Landim AB, Pieroni JP. Ensaios clínicos no Brasil: competitividade internacional e desafios. BNDES Set. 2012;(36):45-84.

26. Cabral MML, Schindler HC, Abath FGC. Regulations, conflicts and ethics of medical research in developing countries. Rev Saude Publica. 2006;40(3):521-7. https://doi.org/10.1590/S0034-89102006000300022

27. Agência Nacional de Vigilância Sanitária - Anvisa. Adendo integrado ao ICH E6(R1): guia de boas práticas clínicas E6(r2). Brasília: Agência Nacional de Vigilância Sanitária; 2019.

28. Shenoy P. Multi-regional clinical trials and global drug development. Perspect Clin Res. 2016;7(2):62-7. https://doi.org/10.4103/2229-3485.179430

29. Thiers FA. The globalization of clinical drug development [dissertação]. Cambridge: Harvard-MIT Division of Health Sciences \& Technology; 2006.

30. Alvarenga LS, Martins EM. Biopharmaceutical industry-sponsor global clinical trials in emerging countries. Rev Assoc Med Bras. 2010;56(4):428-33. https: / /doi.org/10.1590/S0104-42302010000400015

31. Abodor $\mathrm{H}$. Ethical issues in outsourcing: the case of contract medical research and the global pharmaceutical industry. J Bus Ethics. 2012;105(2):239-55. https: / /doi.org/10.1007/s10551-011-0964-0

32. Associação da Indústria Farmacêutica de Pesquisa Interfarma. A importância da pesquisa clínica para o Brasil. São Paulo: Associação da Indústria Farmacêutica de Pesquisa; 2020[acesso jul 2019]. Disponível em: https: / /www. interfarma.org.br/public/files/biblioteca/a-importancia-dapesquisa-clinica-para-o-brasil-interfarma2.pdf

33. Gorski JJ. An FDA-EEC perspective on the international acceptance of foreign clinical data. Cal West Int Law J. 1990;21:1-5.

34. European Medicines Agency - EMA. Reflection paper on the extrapolation of results from clinical studies conducted outside the EU to the EU-population. Amsterdam: European Medicines Agency; 2009.

35. Wechsler J. Research faces challagens in 2010. Applied Clinical Trials. 1 jan 2010.

36. Suarez-Kurtz G, Pena SDJ, Struchiner CJ, Hutz MH. Pharmacogenomic diversity among brazilians: influence of ancestry, self-reported color, and geographical origin. Front Pharmacol. 2012;13:1-7. https://doi.org/10.3389/fphar.2012.00191 
37. Suarez-Kurtz G, Pena SDJ, Hutz MH. Application of the F(ST) statistics to explore pharmacogenomic diversity in the brazilian population. Pharmacogenomics. 2012;13(7):771-7. https://doi.org/10.2217/pgs.12.3

38. Dainesi SM, Goldbaum M. Pesquisa clínica como estratégia de desenvolvimento em saúde. Rev Assoc Med Bras. 2012;58(1):2-6. https://doi.org/10.1590/S0104-42302012000100002

39. Gouy CML, Porto TF, Penido C. Avaliação de ensaios clínicos no Brasil: histórico e atualidades. Rev Bioetica. 2018;26(3):350-9.

https://doi.org/10.1590/1983-80422018263254

40. Santana RS, Leite SN. Prioridades da pesquisa clínica com medicamentos no Brasil e as doenças da pobreza. Rev Panam Salud Publica. 2016;40(5):356-62.

41. Drain PK, Robine M, Holmes KK, Bassett IV. Global migration of clinical trials in the era of trial registration. Nat Rev Drug Discov. 2014;13(3):166-7. https://doi.org/10.1038/nrd4260

Contribuição dos Autores

Silva JCRA - Concepção, planejamento (desenho do estudo), aquisição, análise, interpretação dos dados e redação do trabalho. Silva DLM - Concepção e planejamento (desenho do estudo) e redação do trabalho. Capucho HC, Santana RS - Aquisição, análise, interpretação dos dados e redação do trabalho. Farinasso CM - Redação do trabalho. Todos os autores aprovaram a versão final do trabalho.

Conflito de Interesse

Os autores informam não haver qualquer potencial conflito de interesse com pares e instituições, políticos ou financeiros deste estudo.

Esta publicação está sob a licença Creative Commons Atribuição 3.0 não Adaptada.

Para ver uma cópia desta licença, visite http://creativecommons.org/licenses/by/3.0/deed.pt_BR. 